\title{
Head Injury and Amyotrophic Lateral Sclerosis: A Meta-Analysis
}

\author{
Guodong Liu ${ }^{a}$ Shan Ou ${ }^{b}$ Huijie Cui ${ }^{c}$ Xuan $\mathrm{Li}^{\mathrm{a}}$ Zhiyong Yin ${ }^{d}$ Dongqing $\mathrm{Gu}^{\mathrm{c}}$ \\ Zhengguo Wang ${ }^{d}$
}

aThe Eighth Department, State Key Laboratory of Trauma, Burn and Combined Injuries, Research Institute of Surgery, Daping Hospital, Army Medical University, Chongqing, China; bepartment of Anesthesiology, First People's Hospital of Chengdu, Chengdu, China; 'Department of Epidemiology and Biostatistics, First Affiliated Hospital, Army Medical University, Chongqing, China; ${ }^{\mathrm{T}}$ The Fourth Department, State Key Laboratory of Trauma, Burn and Combined Injuries, Institute for Traffic Medicine, Research Institute of Surgery, Daping Hospital, Army Medical University, Chongqing, China

\section{Keywords}

Head injury · Amyotrophic lateral sclerosis · Risk factor ·

Meta-analysis

\begin{abstract}
Background: Prior studies have suggested that head injury might be a potential risk factor of amyotrophic lateral sclerosis (ALS). However, the association has not been well established. We aimed to provide a synopsis of the current understanding of head injury's role in ALS. Methods: We performed a systematic search in PubMed for observational studies that quantitatively investigated the association between head injury and ALS risk published before April 10, 2020. We used a random-effects model to calculate odds ratios (ORs) and 95\% confidence intervals (Cls). Results: Fourteen eligible articles including 10,703 cases and 2,159,324 controls were selected in current meta-analysis. We found that head injury was associated with an increased risk of ALS (OR $=1.38,95 \% \mathrm{Cl}: 1.20-1.60)$ and the association was slightly stronger concerning severe head injury and ALS risk (OR = 1.69, 95\% Cl: 1.27-2.23). Considering the number of head injuries $(N)$ and ALS risk, the association was weak $(\mathrm{OR}=1.23$, $95 \%$ Cl: $1.10-1.37, N=1$; OR $=1.29,95 \% \mathrm{Cl}: 0.89-1.86, N \geq 2$ ).
\end{abstract}

In addition, a strong association with ALS risk was found in individuals who suffered head injury $<1$ year $(O R=4.05,95 \%$ $\mathrm{Cl}: 2.79-5.89)$, and when the time lag was set at 1-5, 5-10, and $>10$ years, the pooled OR was $1.13,1.35$, and 1.10, respectively. Conclusion: This meta-analysis indicates that head injury, especially severe head injury, could increase ALS risk. Although a strong association is found between head injury $<1$ year and ALS risk in the current study, this result suggests a possibility of reverse causation.

(C) 2021 S. Karger AG, Basel

\section{Introduction}

Head injury is the most frequent cause of nervous tissue damage in developed countries [1]. Annual head injury incidence is in magnitude of millions, and there are $\approx 5.3$ million people in the United States and nearly 7.7 million people in Europe living with head injury-related functional disability [2]. The estimated annual global incidence of head injury requiring medical attention or resulting in hospitalization or death is over 10 million, making it a global health challenge [3]. A possible association of head injury and neurodegenerative diseases was dis-

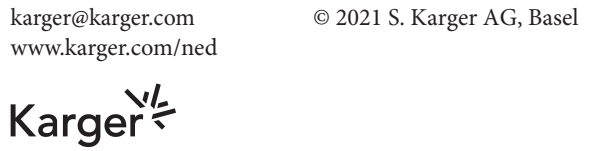


cussed in the early literature, and meta-analyses in recent years further indicate that head injury might be implicated in dementia, Alzheimer's disease, multiple sclerosis, Parkinson's disease (PD), and amyotrophic lateral sclerosis (ALS) [4-8].

ALS is a severe neurodegenerative disease that affects both upper and lower motor neurons, which adversely affects the quality of life of patients and significantly increases the burden of families and society [9]. As a global disease, the overall worldwide prevalence of ALS is $\approx 4.42$ per 100,000 population and the incidence is about 1.59 per 100,000 person-years [10]. The year 2016 witnessed 330,918 new ALS cases which claimed 926,090 disabilityadjusted life years and 34,325 deaths [11]. Though the etiology of ALS remains largely unknown, some existing research studies show that older age, male sex, and family history of ALS have been established as risk factors [12].

The link between history of trauma and ALS risk was reported as early as in 1911 [13]. Since then, this topic has repeatedly been argued for more than a century [14-17]. Some observational studies have suggested that head injury was associated with an increased risk of ALS [14, 15, 17-20], whereas several other studies found no association [1, 16, 21-27]. A few meta-analyses have been performed to investigate this association, however, with inconclusive results. The first meta-analysis by Chen et al. [22] in 2007 included 9 studies and reported an odds ratio (OR) of 1.7. In 2016, a meta-analysis by Perry et al. [4] included 4 studies, demonstrating no evidence for relationship between head injury and ALS risk [4]. Subsequently, Watanabe and Watanabe [8] conducted a metaanalysis involving 16 studies to re-examine the association between the history of head trauma and ALS occurrence. However, the head injury was not specifically defined in some included studies [28-30]. Although the results suggested that head injury was associated with a 1.45 -fold increased ALS risk, conflicting results have been reported recently $[1,16]$. Given the inconsistency in the literature on the role of head injury in risk of ALS, we conducted an up-to-date meta-analysis including recent studies to re-evaluate this association.

\section{Methods}

This meta-analysis was performed according to the PRISMA (Preferred Reporting Items for Systematic Reviews and MetaAnalysis) and MOOSE (Meta-Analysis of Observational Studies in Epidemiology) guidelines [31, 32].

\section{Literature Search}

A comprehensive literature search of related studies was conducted using PubMed (published on or before April 10, 2020), with the following keywords "Amyotrophic Lateral Sclerosis or ALS or Motor Neuron Disease or MND or neurodegenerative disease or Neurological Disease" and "head injury or brain injury or head trauma or brain trauma or traumatic brain injury or brain damage or trauma or craniocerebral trauma or craniocerebral injury" as query terms. The title and abstract of studies, or full text if necessary, were reviewed to identify all relevant publications. In addition, reference lists of all included studies as well as reviews and meta-analyses were manually screened for potential related studies.

\section{Inclusion and Exclusion Criteria}

Inclusion criteria for studies investigating the association between head injury and ALS risk were as follows: (1) case-control, cross-sectional, or cohort studies that quantitatively investigated this association; (2) exposure to head injury (reported as head injury, head trauma, brain injury, brain trauma, traumatic brain injury (TBI), brain damage, craniocerebral trauma, or craniocerebral injury in the original paper) defined based on medical records, military records, questionnaires, or self-reports; (3) the outcome of interest was ALS; (4) providing risk estimates with 95\% confidence intervals (CIs) or data to calculate them (for each study, the risk estimates adjusted for the largest number of variables were extracted; if the multivariate OR was unavailable, we used ageadjusted OR or unadjusted OR; and (5) English language.

When multiple publications use the same study population, we included the most recent publication or publication with largest sample size. Abstracts, reviews, letters, case reports, and studies that did not provide sufficient data to calculate the risk estimates were excluded. Two investigators (G.L. and S.O.) independently selected studies, and any discrepancy was resolved by consensus.

\section{Data Extraction and Quality Assessment}

Data extraction was completed independently by 2 investigators (H.C. and X.L.), and any discrepancy was resolved by the third investigator (D.G.). The following information was extracted from each study: first author, year of publication, country, ethnicity, study design, data source, exposure assessment methods, the number of cases and controls (or sample size for cohort study), OR, relative risk (RR), hazard ratio (HR) or standardized morbidity ratio (SMR) and corresponding 95\% CI, adjusted odds ratio (aOR), adjustments, number of head injuries, year since last injury, and age at head injury.

Quality assessment was performed using the Newcastle-Ottawa Scale (NOS) [33]. Individual studies were assessed based on 3 quality parameters: selection, comparability, and exposure (outcome for cohort study). The maximum total score is 9 , with a score of 7 or greater indicating high study quality.

\section{Statistical Analysis}

Statistical analyses were performed with the use of Stata version 13 (Stata, College Station, TX, USA). Association between head injury and ALS risk was evaluated by OR and corresponding $95 \%$ CI using a random-effects model (DerSimonian Laird) [34]. In addition, meta-analyses considering the time lags since last head injury and the repetition of head injuries relating to the occurrence of ALS were performed. For studies not containing OR, SMR, RR, 


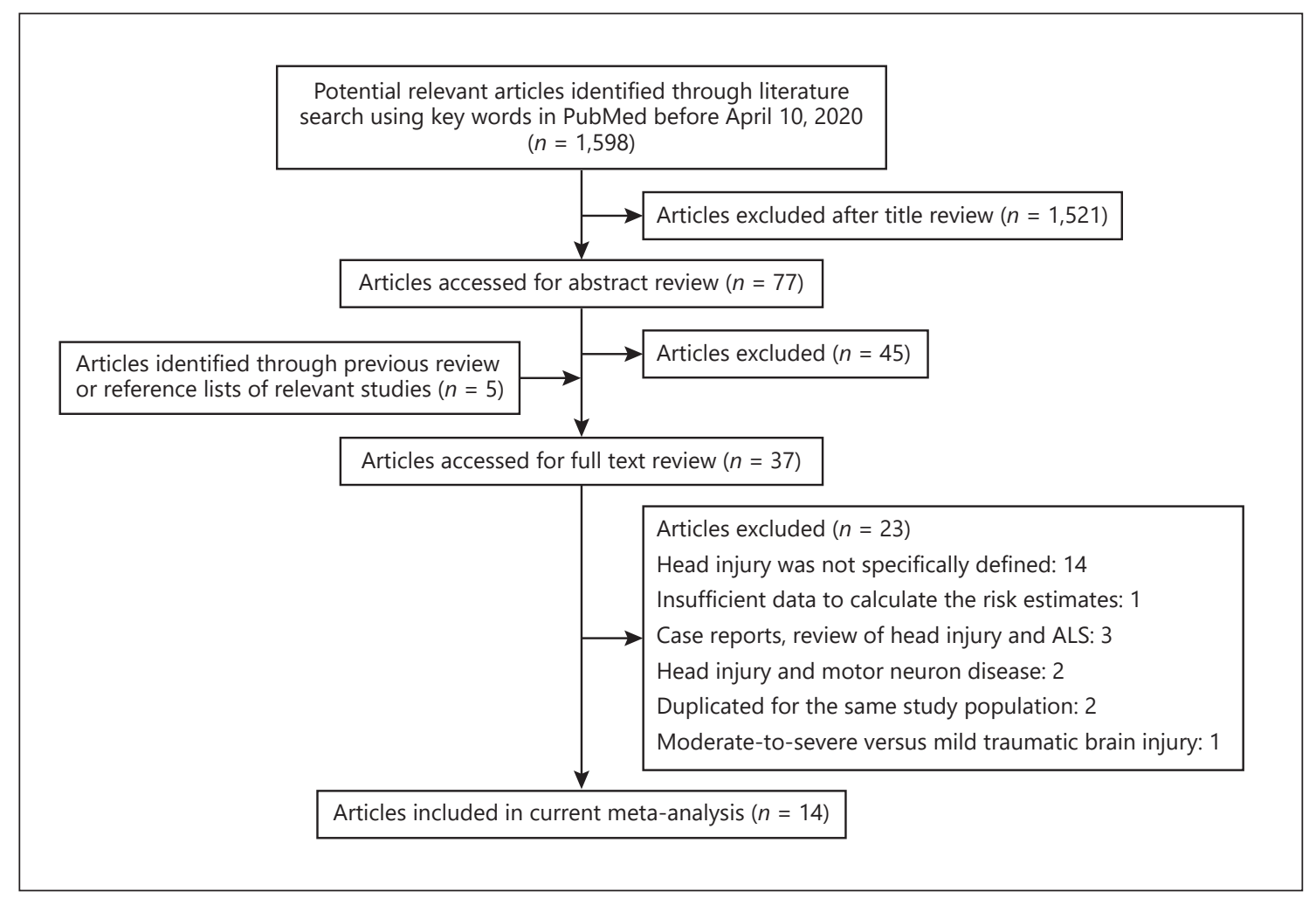

Fig. 1. Flow diagram of literature search and study selection (literature search was made on April 10, 2020).

or HR were treated as substitutes of OR in the current study. If studies did not report the abovementioned statistical outcome, we directly calculated the crude ORs using available original data. If one study had separate ORs for different time lags, we directly merged these ORs using a random-effects model.

Evaluation of meta-analysis included the test of heterogeneity, sensitivity analyses, and publication bias. The heterogeneity among studies was assessed by $I^{2}$ statistic $\left(I^{2}\right.$ values $<25 \%$ represented mild heterogeneity, values between 25 and $50 \%$ represented moderate heterogeneity, and values $>50 \%$ represented large heterogeneity). Subgroup analyses were conducted according to study design (case-control or cohort), exposure assessment methods (medical records or questionnaire/self-report), and severity of head injury (severe or others). In addition, sensitivity analyses were performed to investigate the influence of single studies on the overall risk estimate by removing one study each time. The funnel plot was drawn to inspect potential publication bias, and both Begg's test and Egger's test were used for evaluating funnel plot asymmetry.

\section{Results}

\section{Literature Search Results and Study Characteristics}

The literature search and selection process is presented in Figure 1. The comprehensive search strategy generated
1,598 potentially relevant studies, of which 32 were considered of potential value and the full texts were retrieved for detailed evaluation. In addition, 5 studies were included through previous review or reference lists of relevant studies. We then examined full texts of the remaining 37 studies and excluded 23 of them. To be specific, 14 articles in which head injury was not specifically defined, 3 articles being either case report or review of head injury and ALS, 2 articles about head injury and motor neuron disease, 2 articles using the same study population, 1 article with insufficient data to calculate the risk estimates, and 1 article about moderate-to-severe versus mild TBI were excluded. Finally, 14 studies met our inclusion criteria and were included in the meta-analysis.

The characteristics of the included studies are summarized in Table 1. In total, 10 case-control studies and 4 cohort studies (including 1 nested case-control study) with a total of 10,703 cases and 2,159,324 controls were included. The 10 case-control studies were published between 1980 and 2020 with a total of 6,191 cases and 370,439 controls. Of these studies, 5 studies were conducted in Europe, 5 in USA, and 1 in China. Control subjects in these case-control studies came from a hospital- 


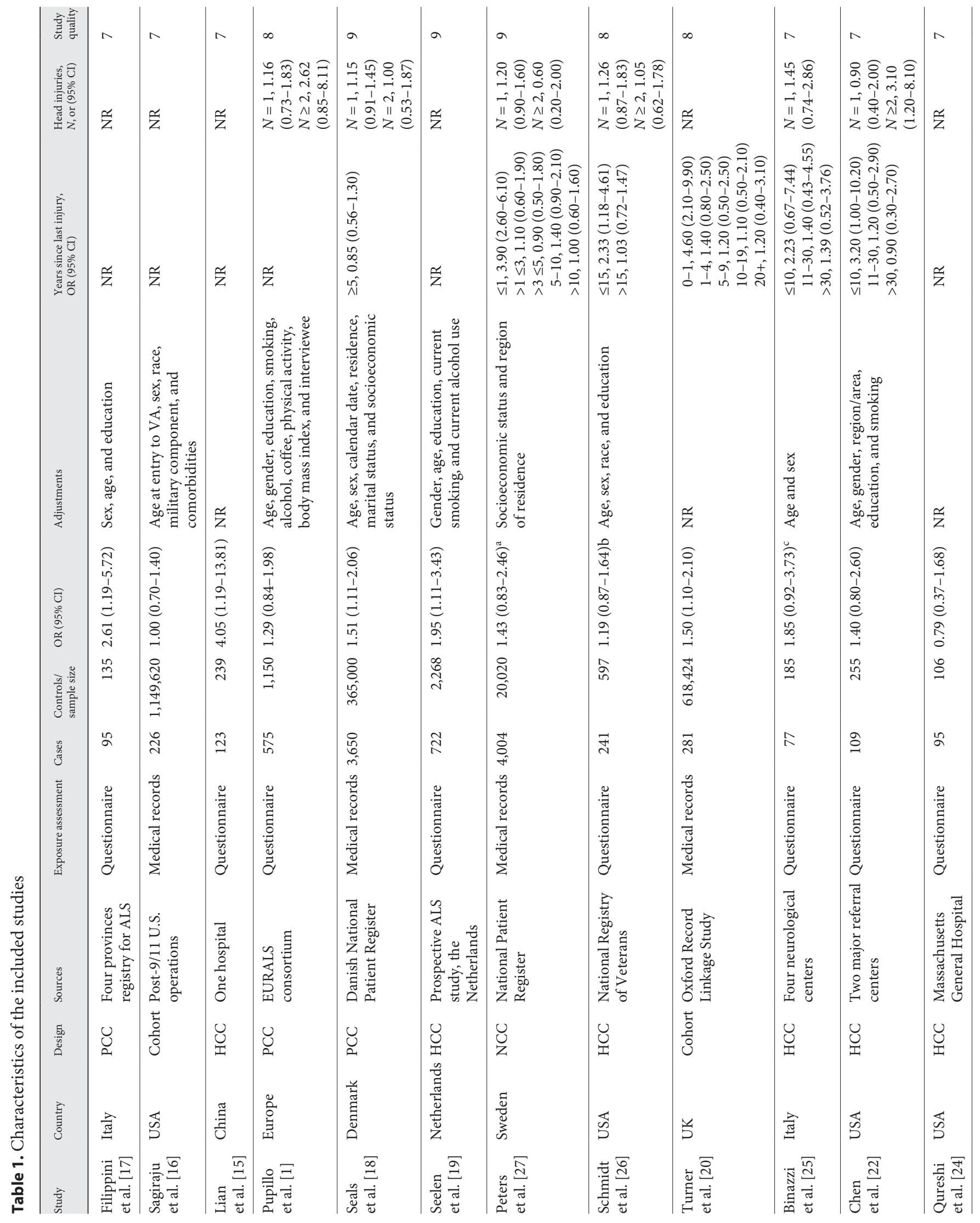




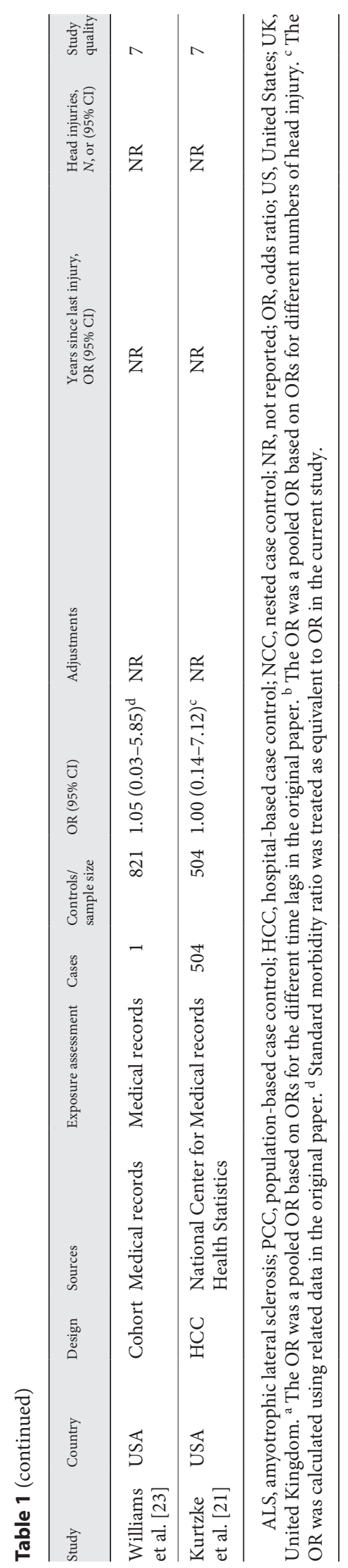

Head Injury and Amyotrophic Lateral Sclerosis based setting in 7 studies and a population-based setting in 3 studies. The 4 cohort studies were published between 1991 and 2019 with a total of 1,788,885 subjects. Of the cohort studies, 2 studies were conducted in USA and 2 in Europe.

In addition, each study included in our meta-analysis was carefully assessed according to NOS. The quality scores ranged from 7 to 9 , which indicated high quality.

\section{Head Injury and the Risk of ALS}

The results of head injury associated with ALS risk are shown in Table 2 and Figure 2. We found a positive association between head injury and ALS risk $(\mathrm{OR}=1.38$, 95\% CI: 1.20-1.60). Subgroup analyses by study design, exposure assessment methods, and severity of the head injury were conducted (Table 2; see online suppl. Fig. 1-3; for all online suppl. material, see www. karger.com/doi/10.1159/000510987). We found that the association between head injury and ALS risk was stronger in case-control studies $(\mathrm{OR}=1.46,95 \% \mathrm{CI}: 1.21-1.76)$ compared with that in cohort studies $(\mathrm{OR}=1.27,95 \% \mathrm{CI}$ : $1.02-1.58)$. The meta-analysis of studies with the exposure assessment methods based on medical records suggested that head injury could increase the risk of ALS (OR $=1.34,95 \%$ CI: 1.12-1.60), whereas studies with the exposure assessment methods based on questionnaire indicated a stronger association $(\mathrm{OR}=1.49,95 \% \mathrm{CI}$ : $1.15-$ 1.92). In addition, the relationship between severe head injury and ALS risk was explored in 4 studies and the results of meta-analysis suggested that severe head injury could increase the risk of ALS (OR $=1.69,95 \%$ CI: 1.27 2.23). However, the association was weaker in other head injury and ALS risk (OR = 1.35, 95\% CI: 1.13-1.61).

Meta-analyses concerning the number of head injuries and time lags since last injury were also performed in current study (Table 3; online suppl. Fig. 4, 5). The association between the number of head injuries and the risk of ALS was explored in 6 studies. The results showed that 1 episode of head injury could increase the risk of ALS $(\mathrm{OR}=1.23,95 \% \mathrm{CI}: 1.10-1.37)$, and when focusing on cases with repeated head injuries, we found a slightly stronger association ( $\mathrm{OR}=1.29,95 \% \mathrm{CI}$ : $0.89-1.86)$.

We then conducted a meta-analysis considering the time lags between the last head injury and the risk of ALS using 5 studies. We found that individuals who suffered head injury for $<1$ year were more likely to be diagnosed with ALS (OR $=4.05,95 \% \mathrm{CI}: 2.79-5.89)$. When the time lag was set at $1-5,5-10$, and $>10$ years, the pooled OR was $1.13,1.35$, and 1.10 , respectively. In addition, we assessed the risk of ALS in individuals with head injuries within 10 
Table 2. Analysis on the role of head injury in ALS risk

\begin{tabular}{|c|c|c|c|c|c|c|c|}
\hline \multirow[t]{2}{*}{ Subgroups } & \multirow[t]{2}{*}{ Studies } & \multirow[t]{2}{*}{ Cases } & \multirow[t]{2}{*}{ Sample size } & \multirow{2}{*}{$\begin{array}{l}\text { Risk estimates } \\
\text { OR (95\% CI) }\end{array}$} & \multirow{2}{*}{$\begin{array}{l}\text { Heterogeneity } \\
I^{2}, \%\end{array}$} & \multicolumn{2}{|c|}{ Publication bias } \\
\hline & & & & & & Begg's $p$ & Egger's $p$ \\
\hline \multicolumn{8}{|l|}{ Study design } \\
\hline Cohort & 4 & 4,512 & $1,792,889$ & $1.27(1.02-1.58)$ & 2 & & \\
\hline Medical records & 6 & 8,666 & $2,162,547$ & $1.34(1.12-1.60)$ & 0 & & \\
\hline Questionnaire & 8 & 2,037 & 6,972 & $1.49(1.15-1.92)$ & 34 & & \\
\hline \multicolumn{8}{|c|}{ Severity of head injury } \\
\hline Severe & 4 & 5,410 & 29,103 & $1.69(1.27-2.23)$ & 0 & & \\
\hline Others & 11 & 5,736 & $2,140,416$ & $1.35(1.13-1.61)$ & 23 & & \\
\hline
\end{tabular}

ALS, amyotrophic lateral sclerosis; CI, confidence interval; OR, odds ratio.

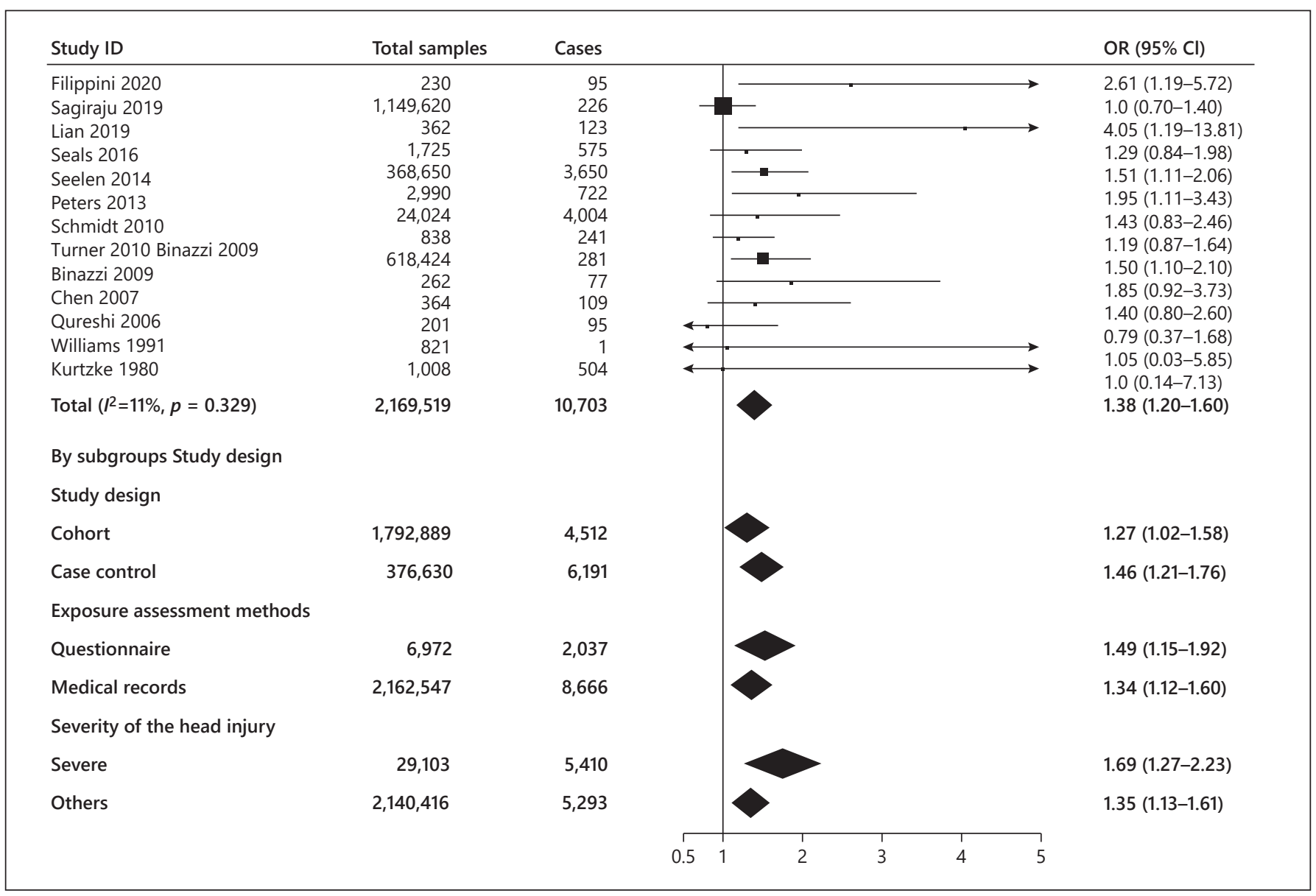

Fig. 2. Forest plots of association between head injury and ALS risk. The square indicates adjusted ORs, the size of the square indicates percentage of the total sample sizes in each study, dashed line indicates the overall estimate of risk, horizontal lines represent 95\% CIs, and diamonds represent the overall ORs. ALS, amyotrophic lateral sclerosis; OR, odds ratio; CI, confidence interval. 
years. Since the clinical symptoms of ALS were not obvious in the first 1 or 2 years, we excluded the individuals who suffered ALS within 1 year of head injury. The results showed that individuals with head injury still had an increased risk of ALS within 10 years $(\mathrm{OR}=1.30,95 \% \mathrm{CI}$ : $1.10-1.54)$.

Heterogeneity, Sensitivity Analysis, and Publication Bias

As shown in Table 2, mild heterogeneity was found among studies concerning relationship between head injury and ALS risk $\left(I^{2}=11 \%\right)$. In addition, sensitivity analysis was performed to evaluate the stability of results (online suppl. Fig. 6). We found that removing any single study could not significantly change the pooled ORs and 95\% CIs. Publication bias was assessed by Begg's funnel plots and Egger's test (Table 2). The funnel plot appeared generally symmetrical (online suppl. Fig. 7). No significant publication bias was detected using either Begg's test or Egger's test in studies concerning head injury and ALS risk (Begg's $p=0.511$; Egger's $p=0.336$ ).

\section{Discussion}

We conducted an up-to-date meta-analysis to re-estimate the relative effect of head injury on ALS risk. In addition, the relationships considering the number of head injuries and the time lags since last head injury with the risk of ALS were evaluated in the current study. To our knowledge, this study is the largest assessment of the literature on association between head injury and ALS risk involving a total of 10,703 cases and 2,159,324 controls. We believe that these findings will provide useful information for clinical decision-making in individuals with head injury.

The present study found that compared with individuals with no history of head injury, those with a history of head injury suffered $\approx 1.38$-fold increased risk of ALS. Furthermore, biological evidence suggested a potential mechanism link between head injury and ALS. Trauma to the head is known to disrupt the blood-brain barrier, which is selectively impermeable to many solutes, including some toxins. It has been hypothesized that deterioration of the barrier might play a role in ALS pathogenesis $[35,36]$. Recent studies have addressed the influence of TBI on disease progression in ALS animal models. In ALS mouse models, TBI can trigger TDP-43 pathology, which is a neuropathological hallmark lesion in brains of patients with ALS [37]. A follow-up study using fly models of ALS found TBI could induce stress granule formation in the brain and might en-

Head Injury and Amyotrophic Lateral Sclerosis hance motor dysfunctions [38]. Since very few studies to date have been performed to explore the pathogenesis in head injury and ALS development, the potential biological mechanism remains unclear [39].

However, because most of the studies investigated individual histories of head injuries using questionnaires, which would give rise to recall bias, the definition of head injury could vary among individuals and the information about the head injury experience could be affected by recall bias. Therefore, we performed a stratified analysis according to exposure assessment methods, and interestingly, the risk estimates were higher in the meta-analysis of studies using questionnaires compared with those using medical records (1.49 vs. 1.34). Moreover, 10 of the 14 studies included in the primary meta-analysis are not cohort but case-control studies and their results might be confounded by various factors such as recall bias and selection bias. Hence, we conducted a meta-analysis containing only the 4 cohort studies and found a weak association between history of head injuries and occurrence of ALS (OR = 1.27). Thus, the current result may likely overestimate this association.

We further found that the associated risk between head injury and ALS increased with severity of head injury. In the current study, individuals suffered severe head injury had a $69 \%$ increased probability of developing ALS. However, a recent cohort study found that moderate-to-severe TBI was only associated with an increased risk for future dementia but not for PD or ALS [40]. Since the absolute number of persons developing ALS was limited in that study, the negative finding might be the consequence of a type II error.

In our study, compared with the individuals who have only 1 episode of head injury, those who suffer repeated head injuries had a slightly higher risk of ALS. There may be a small dose-response relation according to the number of head injuries since the summary OR for repeated injuries was slightly greater (1.29 vs. 1.23$)$. The plausibility of these findings may be that repetitive mild TBI could trigger the development of chronic traumatic encephalopathy (CTE), a progressive neurodegeneration linked to repetitive head impacts and has been associated with ALS $[41,42]$. CTE is characterized by the widespread deposition of TDP-43 and tau which has also been observed in neuropathological hallmarks of ALS disease [43, 44]. Otherwise, the incidence and mortality of ALS were unusually high among professional soccer players and American football players [45-48]. Interestingly, TDP-43 pathology was shown in the brains of athletes (boxing and football) who had repetitive head injuries $[49,50]$.

Neuroepidemiology 2021;55:11-19 DOI: $10.1159 / 000510987$ 
In addition, the time lags between the last head injury and the ALS occurrence were taken into consideration in the current study, and we found that individuals who suffered head injury $<1$ year were more likely to be diagnosed with ALS. This result should be interpreted with caution because of possible reverse causation. The clinical symptoms of ALS were usually not obvious in the first 1 or 2 years, and head injury $<1$ year may be an early sign of ALS [20]. Therefore, head injury that occurs within 1 year of formal diagnosis of ALS may simply reflect the early motor impairments of undiagnosed ALS, rather than trigger its onset [39]. In addition, current findings of the time-lag analyses suggested that very old trauma (defined as trauma that occurred at least 10 years before ALS) had little association with ALS risk, which reflected a possibility of reverse causation as well. In the meantime, we should note that recall bias caused by cognitive impairments in ALS may also account for the results. Since many of the included studies here investigated individual histories of head injuries using questionnaires and ALS patients who had more difficulty in recalling their memories of old traumas due to cognitive impairments than the controls, the association between the history of old head injuries and the occurrence of ALS might be underestimated [8].

Several limitations in this meta-analysis should be considered. First, although we have tried our best to contact with the corresponding author for the original data, we excluded some related studies in meta-analysis due to insufficient data to calculate the risk estimates. Second, although we extracted the most fully adjusted risk estimates, adjusted confounders varied among the included studies and some potential confounding factors (such as age, sex, race, smoking status, and alcohol consumption) may influence the relationship between head injury and ALS risk. Third, meta-analysis on time lag could also be affected by the scarcity of studies and several residual confounding factors compared with the primary analysis. Fourth, some of the included studies were based on hospital-based datasets, which would potentially induce selection bias. Finally, the methods used to assess traumat- ic events and injuries may be imprecise because of lack of standard definitions of exposures.

In conclusion, the findings from this meta-analysis indicate that head injury especially severe head injury could increase the risk of ALS. In addition, we found that compared with the individuals who have only 1 episode of head injury, those who suffer repeated head injuries had a slightly higher risk of ALS. Although the results suggest individuals who have head injury for $<1$ year are more likely to develop ALS, this result should be interpreted with caution because of possible reverse causation. Future well-designed case-control or cohort studies considering the time lags between the occurrence of head injuries and the diagnosis of ALS are warranted to confirm the role of head injury in the pathogenesis of ALS.

\section{Acknowledgements}

The authors sincerely thank all the subjects who participated in this study.

\section{Conflict of Interest Statement}

The authors have no conflicts of interest to declare.

\section{Funding Sources}

This work was supported by the China Postdoctoral Science Foundation (2014M562594 and 2015T81090) and Postdoctoral Science Foundation of Chongqing (Xm2015034).

\section{Author Contributions}

Z.W. and D.G. designed the study and obtained financial support. G.L., S.O., H.C., and X.L. conducted the literature search and data extraction and management. Z.Y. performed statistical analysis and drew plots. G.L. wrote the first draft of the original manuscript with significant contributions from S.O., H.C., X.L., Z.Y., D.G., and Z.W. All authors have reviewed and approved the final manuscript.

\section{References}

1 Pupillo E, Poloni M, Bianchi E, Giussani G, Logroscino G, Zoccolella S, et al. Trauma and amyotrophic lateral sclerosis: a European population-based case-control study from the EURALS consortium. Amyotroph Lateral Scler Frontotemporal Degener. 2018 Feb; 19(1-2):118-25.

2 Rubiano AM, Carney N, Chesnut R, Puyana JC. Global neurotrauma research challenges and opportunities. Nature. 2015 Nov; 527(7578):S193-7.

3 Hyder AA, Wunderlich CA, Puvanachandra P, Gururaj G, Kobusingye OC. The impact of traumatic brain injuries: a global perspective. Neurorehabilitation. 2007 Jan;22(5): 341-53.

4 Perry DC, Sturm VE, Peterson MJ, Pieper CF, Bullock T, Boeve BF, et al. Association of traumatic brain injury with subsequent neurological and psychiatric disease: a metaanalysis. J Neurosurg. 2016 Feb;124(2):51126.

5 Lunny CA, Fraser SN, Knopp-Sihota JA. Physical trauma and risk of multiple sclerosis: a systematic review and meta-analysis of observational studies. J Neurol Sci. 2014 Jan; 336(1-2):13-23.
18

Neuroepidemiology 2021;55:11-19 DOI: $10.1159 / 000510987$
Liu/Ou/Cui/Li/Yin/Gu/Wang 
6 Li Y, Li Y, Li X, Zhang S, Zhao J, Zhu X, et al. Head injury as a risk factor for dementia and Alzheimer's disease: a systematic review and meta-analysis of 32 observational studies. PLoS One. 2017 Jan;12(1):e0169650.

7 Jafari S, Etminan M, Aminzadeh F, Samii A. Head injury and risk of Parkinson disease: a systematic review and meta-analysis. Mov Disord. 2013 Aug;28(9):1222-9.

8 Watanabe Y, Watanabe T. Meta-analytic evaluation of the association between head injury and risk of amyotrophic lateral sclerosis. Eur J Epidemiol. 2017 Oct;32(10):867-79.

9 Valadi N. Evaluation and management of amyotrophic lateral sclerosis. Prim Care. 2015 Jun;42(2):177-87.

10 Xu L, Liu T, Liu L, Yao X, Chen L, Fan D, et al. Global variation in prevalence and incidence of amyotrophic lateral sclerosis: a systematic review and meta-analysis. J Neurol. 2020 Apr;267(4):944-53.

11 GBD 2016 Motor Neuron Disease Collaborators. Global regional, and national burden of motor neuron diseases 1990-2016: a systematic analysis for the global burden of disease study 2016. Lancet Neurol. 2018 Dec;17(12):1083-97.

12 Ingre C, Roos PM, Piehl F, Kamel F, Fang F. Risk factors for amyotrophic lateral sclerosis. Clin Epidemiol. 2015 Jan;7:181-93.

13 Woods AH. Trauma as a cause of amyotrophic lateral sclerosis. JAMA. 1911;LVI(No. 25):1876-7.

14 Tsai CP, Hu C, Lee CT. Finding diseases associated with amyotrophic lateral sclerosis: a total population-based case-control study. Amyotroph Lateral Scler Frontotemporal Degener. 2019 Feb;20(1-2):82-9.

15 Lian L, Liu M, Cui L, Guan Y, Liu T, Cui B, et al. Environmental risk factors and amyotrophic lateral sclerosis (ALS): a case-control study of ALS in China. J Clin Neurosci. 2019 Aug;66:12-8.

16 Sagiraju HKR, Živković S, VanCott AC, Patwa H, Gimeno Ruiz de Porras D, Amuan ME, et al. Amyotrophic lateral sclerosis among veterans deployed in support of post-9/11 U.S. conflicts. Mil Med. 2020 Mar;185(3-4):e501-9.

17 Filippini T, Fiore M, Tesauro M, Malagoli C, Consonni M, Violi F, et al. Clinical and lifestyle factors and risk of amyotrophic lateral sclerosis: a population-based case-control study. Int J Environ Res Public Health. 2020 Jan;17(3):17.

18 Seals RM, Hansen J, Gredal O, Weisskopf MG. Physical trauma and amyotrophic lateral sclerosis: a population-based study using Danish national registries. Am J Epidemiol. 2016 Feb;183(4):294-301.

19 Seelen M, van Doormaal PT, Visser AE, Huisman MH, Roozekrans MH, de Jong SW, et al. Prior medical conditions and the risk of amyotrophic lateral sclerosis. J Neurol. 2014 Oct; 261(10):1949-56.

20 Turner MR, Abisgold J, Yeates DG, Talbot K, Goldacre MJ. Head and other physical trauma requiring hospitalisation is not a significant risk factor in the development of ALS. J Neurol Sci. 2010 Jan;288(1-2):45-8.
21 Kurtzke JF, Beebe GW. Epidemiology of amyotrophic lateral sclerosis: 1. a case-control comparison based on ALS deaths. Neurology. 1980 May;30(5):453-62..0

22 Chen H, Richard M, Sandler DP, Umbach DM, Kamel F. Head injury and amyotrophic lateral sclerosis. Am J Epidemiol. 2007 Oct; 166(7):810-6

23 Williams DB, Annegers JF, Kokmen E, O'Brien PC, Kurland LT. Brain injury and neurologic sequelae: a cohort study of dementia, parkinsonism, and amyotrophic lateral sclerosis. Neurology. 1991 Oct;41(10):1554-7.

24 Qureshi MM, Hayden D, Urbinelli L, Ferrante K, Newhall K, Myers D, et al. Analysis of factors that modify susceptibility and rate of progression in amyotrophic lateral sclerosis (ALS). Amyotroph Lateral Scler. 2006 Sep;7(3):173-82.

25 Binazzi A, Belli S, Uccelli R, Desiato MT, Talamanca IF, Antonini G, et al. An exploratory case-control study on spinal and bulbar forms of amyotrophic lateral sclerosis in the province of Rome. Amyotroph Lateral Scler. 2009 Oct; 10(5-6):361-9.

26 Schmidt S, Kwee LC, Allen KD, Oddone EZ Association of ALS with head injury, cigarette smoking and APOE genotypes. J Neurol Sci. 2010 Apr;291(1-2):22-9.

27 Peters TL, Fang F, Weibull CE, Sandler DP, Kamel F, Ye W. Severe head injury and amyotrophic lateral sclerosis. Amyotroph Lateral Scler Frontotemporal Degener. 2013 May; 14(4):267-72.

28 Kondo K, Tsubaki T. Case-control studies of motor neuron disease: association with mechanical injuries. Arch Neurol. 1981 Apr; 38(4):220-6.

29 Deapen DM, Henderson BE. A case-control study of amyotrophic lateral sclerosis. Am J Epidemiol. 1986 May;123(5):790-9.

30 Gallagher JP, Sanders M. Trauma and amyotrophic lateral sclerosis: a report of 78 patients. Acta Neurol Scand. 1987 Feb;75(2):145-50.

31 Stroup DF, Berlin JA, Morton SC, Olkin I, Williamson GD, Rennie D, et al. Meta-analysis of observational studies in epidemiology: a proposal for reporting. Meta-analysis of Observational Studies in Epidemiology (MOOSE) group. JAMA. 2000 Apr;283(15):2008-12.

32 Moher D, Liberati A, Tetzlaff J, Altman DG. Preferred reporting items for systematic reviews and meta-analyses: the PRISMA statement. BMJ. 2009 Jul;339:b2535.

33 Stang A. Critical evaluation of the NewcastleOttawa scale for the assessment of the quality of nonrandomized studies in meta-analyses. Eur J Epidemiol. 2010 Sep;25(9):603-5.

34 Higgins JP, Thompson SG. Quantifying heterogeneity in a meta-analysis. Stat Med. 2002 Jun;21(11):1539-58.

35 Zlokovic BV. The blood-brain barrier in health and chronic neurodegenerative disorders. Neuron. 2008 Jan;57(2):178-201.

36 Shlosberg D, Benifla M, Kaufer D, Friedman A. Blood-brain barrier breakdown as a therapeutic target in traumatic brain injury. Nat Rev Neurol. 2010 Apr;6(7):393-403.
37 Wiesner D, Tar L, Linkus B, Chandrasekar A Olde Heuvel F, Dupuis L, et al. Reversible induction of TDP-43 granules in cortical neurons after traumatic injury. Exp Neurol. 2018 Jan;299(Pt A):15-25.

38 Anderson EN, Gochenaur L, Singh A, Grant R, Patel K, Watkins S, et al. Traumatic injury induces stress granule formation and enhances motor dysfunctions in ALS/FTD models. Hum Mol Genet. 2018 Apr;27(8):1366-81.

39 Franz CK, Joshi D, Daley EL, Grant RA, Dalamagkas K, Leung A, et al. Impact of traumatic brain injury on amyotrophic lateral sclerosis: from bedside to bench. J Neurophysiol. 2019 Sep;122(3):1174-85.

40 Raj R, Kaprio J, Korja M, Mikkonen ED, Jousilahti P, Siironen J. Risk of hospitalization with neurodegenerative disease after moderate-tosevere traumatic brain injury in the workingage population: a retrospective cohort study using the Finnish national health registries. PLoS Med. 2017 Jul;14(7):e1002316.

41 VanItallie TB. Traumatic brain injury (TBI) in collision sports: possible mechanisms of transformation into chronic traumatic encephalopathy (CTE). Metab Clin Exp. 2019 Nov;100S: 153943.

42 Walt GS, Burris HM, Brady CB, Spencer KR, Alvarez VE, Huber BR, et al. Chronic traumatic encephalopathy within an amyotrophic lateral sclerosis brain bank cohort. J Neuropathol Exp Neurol. 2018 Dec;77(12):1091-100.

43 McKee AC, Cantu RC, Nowinski CJ, HedleyWhyte ET, Gavett BE, Budson AE, et al. Chronic traumatic encephalopathy in athletes: progressive tauopathy after repetitive head injury. J Neuropathol Exp Neurol. 2009 Jul;68(7):709-35.

44 McKee AC, Stern RA, Nowinski CJ, Stein TD, Alvarez VE, Daneshvar DH, et al. The spectrum of disease in chronic traumatic encephalopathy. Brain. 2013 Jan;136(Pt 1):43-64.

45 Lehman EJ, Hein MJ, Baron SL, Gersic CM. Neurodegenerative causes of death among retired national football league players. Neurology. 2012 Nov;79(19):1970-4.

46 Beretta S, Carri MT, Beghi E, Chiò A, Ferrarese $\mathrm{C}$. The sinister side of Italian soccer. Lancet Neurol. 2003 Nov;2(11):656-7.

47 Chio A, Benzi G, Dossena M, Mutani R, Mora G. Severely increased risk of amyotrophic lateral sclerosis among Italian professional football players. Brain. 2005 Mar;128(Pt 3):472-6.

48 Belli S, Vanacore N. Proportionate mortality of Italian soccer players: is amyotrophic lateral sclerosis an occupational disease? Eur J Epidemiol. 2005 Jan;20(3):237-42.

49 Johnson VE, Stewart W, Trojanowski JQ, Smith DH. Acute and chronically increased immunoreactivity to phosphorylation-independent but not pathological TDP-43 after a single traumatic brain injury in humans. Acta Neuropathol. 2011 Dec;122(6):715-26.

50 McKee AC, Gavett BE, Stern RA, Nowinski CJ, Cantu RC, Kowall NW, et al. TDP-43 proteinopathy and motor neuron disease in chronic traumatic encephalopathy. J Neuropathol Exp Neurol. 2010 Sep;69(9):918-29. 